\title{
Evaluation of Touch Trigger Probe Measurement Uncertainty Using FEA
}

\author{
Mohd Rizal Salleh, Qing Ping Yang, Barry Jones \\ School of Engineering and Design, Brunel University, Middlesex UB8 3PH, UK \\ Phone: +44 1895 265854, Fax: +44 1895 269763, Email: emstppy@brunel.ac.uk
}

\begin{abstract}
Evaluation of measurement uncertainty is an essential subject in dimensional measurement. It has also become a dominant issue in Coordinate Measuring Machine (CMM) even though its machine performance has been well accepted by many users. CMM probes, especially touch trigger probes which are commonly used, have been acknowledged as a key error source, largely due to pretravel variations. The probe errors result in large measurement uncertainty in CMM measurement. Various methods have been introduced to estimate measurement uncertainty, but they tend to be time consuming and necessarily require a large amount of experimental data for analyzing the uncertainty.

This paper presents the method of evaluation of CMM probe uncertainty using FEA modeling. It is started with the investigation of the behavior of probe by recording stylus displacement with vary triggering force. Then, those displacement results will be analyzed with sensitivity analysis technique to estimate the uncertainty of recorded results.
\end{abstract}

Keywords - Evaluation of Measurement Uncertainty, Coordinate Measuring Machine, Touch Trigger Probe, Pre-travel Variation, Measurement Uncertainty, Finite Elements Analysis (FEA)

\section{INTRODUCTION}

Whilst uncertainty evaluation has been increasingly applied in various measurements, evaluation of CMM measurement uncertainties still remains practically difficult. It is dominantly due to the complexities of the machine with a large number of error sources affecting the accuracy of measurement results. There has been some progress in the evaluation of the uncertainty of CMM hardware. For instance, factorial design technique has been used in [1] for estimating the CMM uncertainty [2].

Many factors have been identified as the source of measurement errors and uncertainties in CMM. One of them is probe system and it has a critical role in the CMM measurement, not only in terms of its functionality, but also its contribution towards the overall measurement error and uncertainty. Probe pre-travel variation is a significant error in CMM measurement. It has been widely discussed in the literature and a number of ideas have been proposed to reduce the probe errors. However, little research has been reported about the uncertainty evaluation of CMM probes.

Evaluation of measurement uncertainty generally starts with the mathematical model of the measurement system and process [3]. Since CMM probe operation is influenced by many input quantities such as probing angle, probe orientation, stylus configuration, stylus materials, environment, work piece conditions, etc. [3-4], it is very difficult to analytically derive the mathematical model. One alternative method is to formulate the model experimentally. However, this will be very time consuming and costly to implement. This paper will present the method and results of using FEA for the evaluation of CMM probe measurement uncertainty. Section 2 will present the problem and model construction of probe system using FEA. Section 3 presents and discusses the results of modeled pre-travel variation at different probe configurations, orientations and approach directions. It also descries the probe uncertainty evaluation based upon the constructed model. The standard uncertainty of each parameter and its sensitivity coefficient are first determined and then combined following the GUM [5]. The expanded uncertainty and its interpretation with the modeled results are also presented.

\section{PROBLEM DEFINITION AND MODEL CONSTRUCTION}

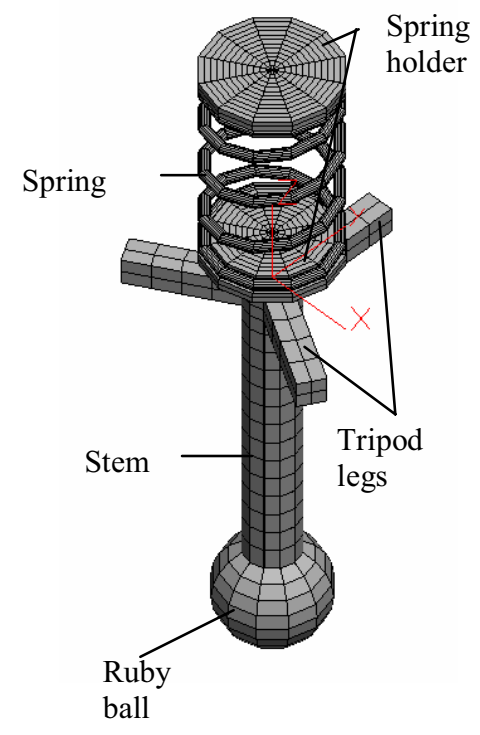

Fig.1: A FEA model of TP2-5W Renishaw's probe system

Although probe manufacturers have carried out some FEA analysis on some of their probe models, the results have not been published for commercial reasons. In fact, it has been very hard to obtain the right probe design data for the same reason. The methodology we adopted is to start with simplified FEA modeling of the probe system and then add details to refine the model. Of course, in doing so the essential characteristics of the probe must be modeled, including analysis of a number of important parameters. A 
Renishaw's TP2-5W probe had been dismantled and the dimensions of some required components were measured for constructing this model. This probe has been chosen as a model because it is commonly utilized and well accepted in industries.

The FEA model generally consists of a stylus and probe system. The probe system is composed of a pair of spring holder, a compression spring and stylus holder with a tripod legs. Then, a stylus with a ruby's ball tip is attached to the stylus holder. Based on our first model in [6], the structure of probe system has been refined by replacing the hollow cylinder with the spring and a range of triggering forces have been applied to trigger the probe (corresponding to constant triggering resistance in the probe circuits). Fig. 1 illustrated the constructed FEA model of TP2-5W probe system which is modeled with the FEA software. As depicted in Fig. 1, each of the tripod's leg has a length of $6 \mathrm{~mm}$ from probe's center. Those legs rest on three pairs of steel balls. One or two legs could be a fulcrum or switch when the stylus is in contact with the work piece. In this model, the boundary conditions were applied at the appropriate legs depending upon the direction of the triggering force. Therefore, several models are required to model the operation where a range of triggering forces with different approach directions is applied. The compression spring used in this probe has a free length of $8 \mathrm{~mm}$, a wire diameter of $0.5 \mathrm{~mm}$ and an outer diameter of $6 \mathrm{~mm}$ with 3 active coils.

Several stylus lengths ranging from 7 to $50 \mathrm{~mm}$ have been chosen for investigating individual stylus errors and uncertainty. Due to lack of information, some assumptions were made on the materials properties, whist a few of them can be obtained from catalogue provided by manufacturer. The material properties of probe system used in our FEA modeling are given in Table 1 below. However, different combination of materials can also be tested. This is the advantage of FEA modeling.

Table 1: TP2-5W Probe Model Materials Properties

\begin{tabular}{cccc}
\hline Materials & $\begin{array}{c}\text { Density } \\
\left(\mathrm{g} / \mathrm{cm}^{3}\right)\end{array}$ & $\begin{array}{c}\text { Young } \\
\left(\mathrm{kgf} / \mathrm{cm}^{2}\right)\end{array}$ & Poisson \\
\hline \hline Tungsten & 15.8 & $4.59 \times 10^{9}$ & 0.24 \\
Stainless steel & 7.9 & $2.039 \times 10^{9}$ & 0.28 \\
Ruby & 3.96 & $4.487 \times 10^{9}$ & 0.3 \\
Bakelite & 2.14 & $5.1 \times 10^{6}$ & 0.33 \\
\hline
\end{tabular}

Due to the symmetry of the model and loading, only $1 / 6$ of the probing directions need to be analyzed. Vary triggering forces have been applied normal to stylus ball tip. The magnitude of forces has been calculated from equation (1) from [7].

The minimum force required to trigger the probe, $F_{m}$, is 7 g.f and this amount of force has been recommended by manufacturer to lift up the tripod legs.

$$
F=\frac{R F_{m}}{p} \cdot \frac{1}{\sin \theta \cos \left(\varphi-\varphi_{0}\right)+\beta \cos \theta}
$$

As stated earlier, due to the symmetrical shape of probe system, only part of this structure will be analyzed. Force will be applied at three different positions around the stylus ball tip with a $30^{\circ}$ interval. The distance between latitudes is $45^{\circ}$. Then, the interpolation method is used to generate more results of pre-travel variation.

\section{RESULTS}

This study principally investigates the behavior of probe system by varying several parameters to study their effects on measurement errors and uncertainty, including sensitivity analysis and the combined standard uncertainty.

Table 2 presents the results for stylus displacement at three different stylus lengths when it is approached at the equator with variable triggering forces.

Table 2: Displacement results for different stylus lengths

\begin{tabular}{cccc}
\hline \multirow{2}{*}{ Index $\left({ }^{\circ}\right)$} & \multicolumn{3}{c}{ Stylus displacement $(\mathrm{mm})$} \\
\cline { 2 - 4 } & $7 \mathrm{~mm}$ & $20 \mathrm{~mm}$ & $50 \mathrm{~mm}$ \\
\hline \hline 0 & 0.108 & 0.312 & 1.215 \\
20 & 0.105 & 0.309 & 1.208 \\
30 & 0.103 & 0.305 & 1.202 \\
40 & 0.100 & 0.302 & 1.195 \\
50 & 0.099 & 0.299 & 1.182 \\
60 & 0.098 & 0.296 & 1.169 \\
70 & 0.097 & 0.292 & 1.182 \\
80 & 0.098 & 0.296 & 1.169 \\
90 & 0.099 & 0.299 & 1.182 \\
100 & 0.100 & 0.302 & 1.195 \\
110 & 0.103 & 0.305 & 1.202 \\
120 & 0.105 & 0.309 & 1.208 \\
130 & 0.108 & 0.312 & 1.215 \\
140 & 0.105 & 0.309 & 1.208 \\
150 & 0.103 & 0.305 & 1.202 \\
160 & 0.100 & 0.302 & 1.195 \\
170 & 0.099 & 0.299 & 1.182 \\
180 & 0.098 & 0.296 & 1.169 \\
\hline
\end{tabular}

From the above results, it is clear that the stylus length has a great impact on the stylus displacement. The results indicate that the displacement recorded decreased from index $0^{\circ}$ to $60^{\circ}$ and rose up from $70^{\circ}$ to $120^{\circ}$. When plotted these results will produce lobing patterns. As shown in Fig. 2, a typical pattern has three lobes, corresponding to the variation of displacement due to the existing of tripod legs structure in the probe system. 


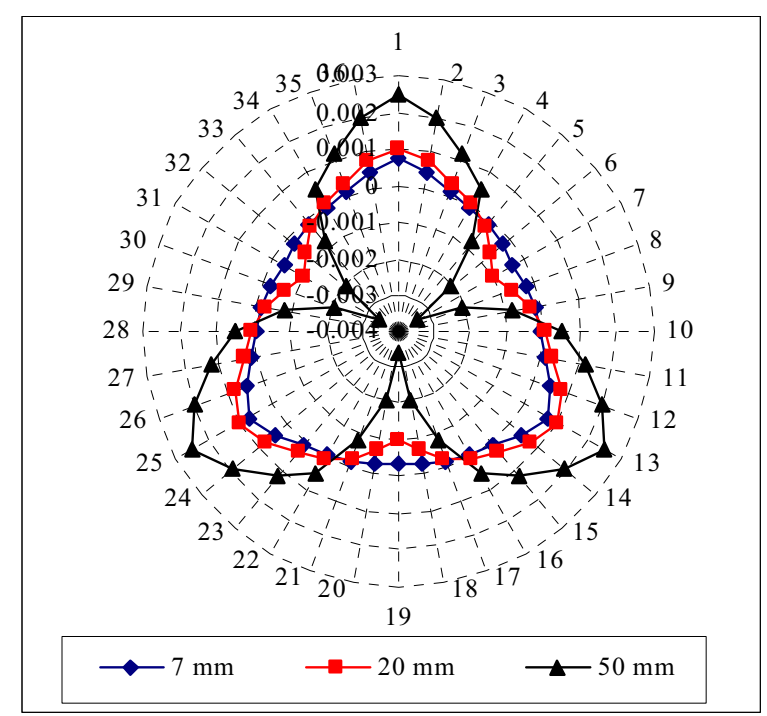

Fig. 2: Variation of pre-travel when stylus being approached perpendicular to the surface at the equator

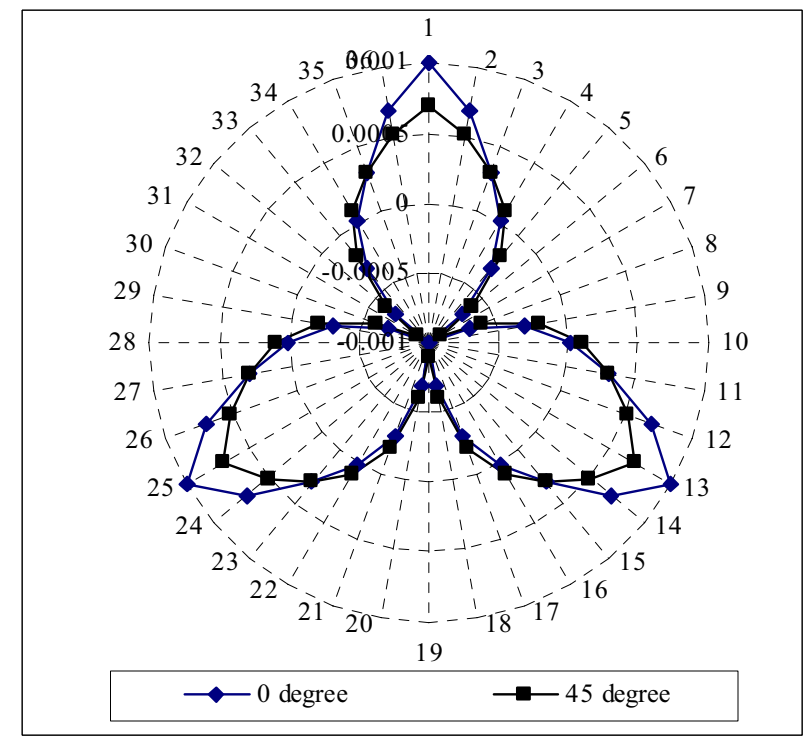

Fig. 3: Displacement at two different latitudes with the similar stylus length $20 \mathrm{~mm}$

Further investigations have also been performed to look into the change of probe lobing patterns when the approach directions are made at different latitudes. Approach direction was made normal to the surface of stylus ball tip, with the stylus length remaining unchanged. From Fig. 3, it can be seen that larger errors are recorded at the equator (at 0 degree).

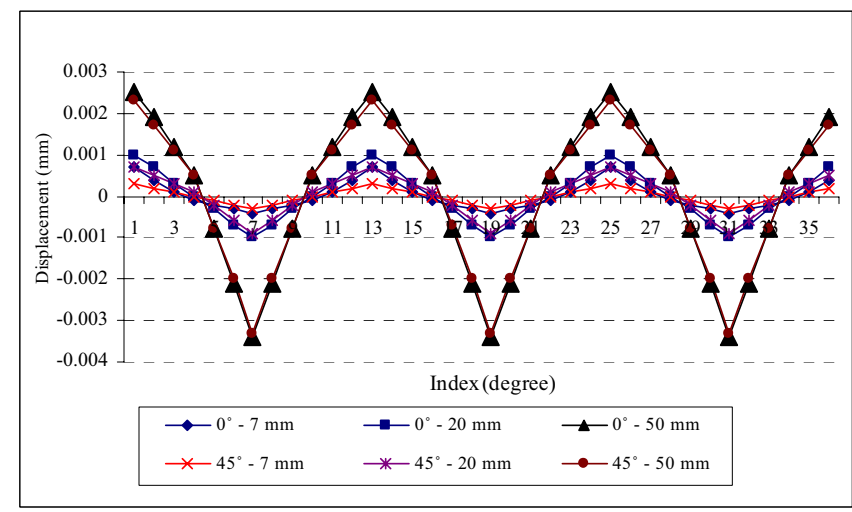

Fig. 4: Displacement results of vary stylus at different latitude

From the data generated from the modeling, the probe measurement uncertainty can be evaluated. The probe displacement or probe measurement error is a function of a number of parameters, three of which were initially considered, i.e. the probe length $L$, latitude angle $\theta$ and probing angle $\varphi$ :

$$
e=f(L, \theta, \varphi)
$$

The uncertainties in $L$ and $\theta$ at each $\varphi$ are calculated using type $\mathrm{B}$ evaluation. A uniform distribution is assumed for both $\mathrm{L}$ and $\theta$, with $\mathrm{L}$ ranging from 7 to $50 \mathrm{~mm}$ and $\theta$ from 0 to 45 degree. The standard uncertainties are therefore given by:

$$
\begin{aligned}
& U_{L}(\varphi)=\frac{43}{\sqrt{3}} \\
& U_{\theta}(\varphi)=\frac{45}{\sqrt{3}}
\end{aligned}
$$

Next, the sensitivity coefficient of each variables, $L$ and $\theta$, is estimated from the modeled results. They are computed using the simulated errors of individual variables according to the equations (5) and (6), respectively.

$C_{L}(\varphi)=\frac{\partial e}{\partial L}=\frac{1}{2} \cdot\left(\frac{e_{50}-e_{20}}{30}+\frac{e_{20}-e_{7}}{13}\right)$

$C_{\theta}(\varphi)=\frac{\partial e}{\partial \theta}=\frac{e_{45^{\circ}}-e_{0^{o}}}{45}$

The overall combined uncertainty in the result can be obtained by combining the individual contributions as follows:

$$
U_{c}(\varphi)=\sqrt{\left(C_{\theta}(\varphi) U_{\theta}(\varphi)\right)^{2}+\left(C_{L}(\varphi) U_{L}(\varphi)\right)^{2}}
$$


Finally, the expanded uncertainty, $U_{p}(\varphi)$, can be obtained at a confidence level of $95 \%$ :

$$
U_{p}(\varphi)=1.96 U_{c}(\varphi)
$$

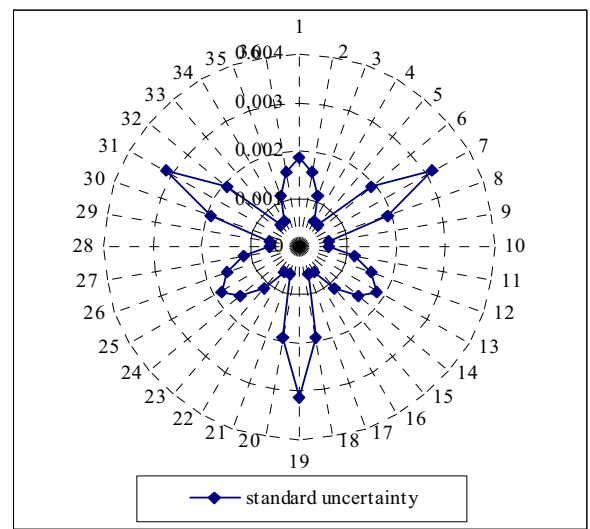

Fig. 5: Computed standard uncertainty, $U_{c}(\varphi)$ for TP2-5W touch trigger probe

By using equation (3) to (6), the probe uncertainties at $\varphi$ due to $L$ and $\theta$ were computed. These individual uncertainties were then combined together using equation (7) and the results are plotted in Fig. 5. It shows that larger uncertainties occurred at the angular positions of $60^{\circ}, 180^{\circ}$ and $300^{\circ}$.

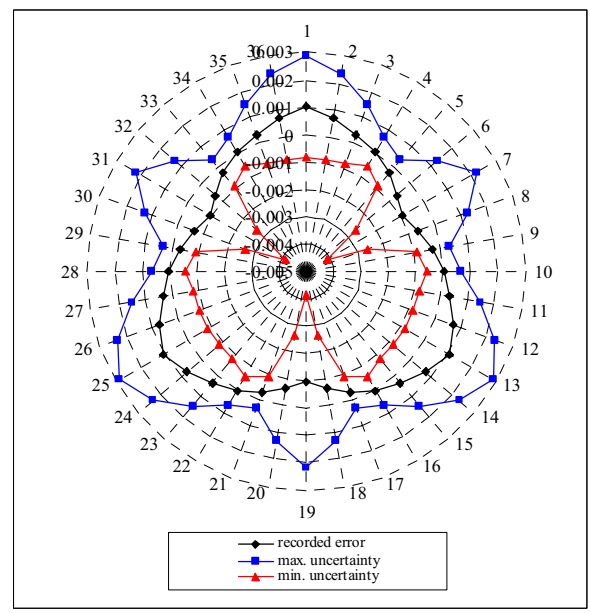

Fig. 6: Probe measurement uncertainty (stylus length $=20 \mathrm{~mm}$, latitude $=0^{\circ}$ at the equator)

Using the expanded uncertainty $U_{p}(\varphi)$, the error range for a single stylus length at the equator can be produced. As shown in Fig. 6, for $20 \mathrm{~mm}$ stylus the maximum and minimum uncertainty value computed are $6.27 \mu \mathrm{m}$ and $1.23 \mu \mathrm{m}$ respectively. This uncertainty linearly increases with stylus length used for the measurement operation.

The probe uncertainties can be similarly combined with the results under other conditions. Fig. 7 shows the probe uncertainty with a stylus length of $20 \mathrm{~mm}$ and a latitude of $45^{\circ}$.

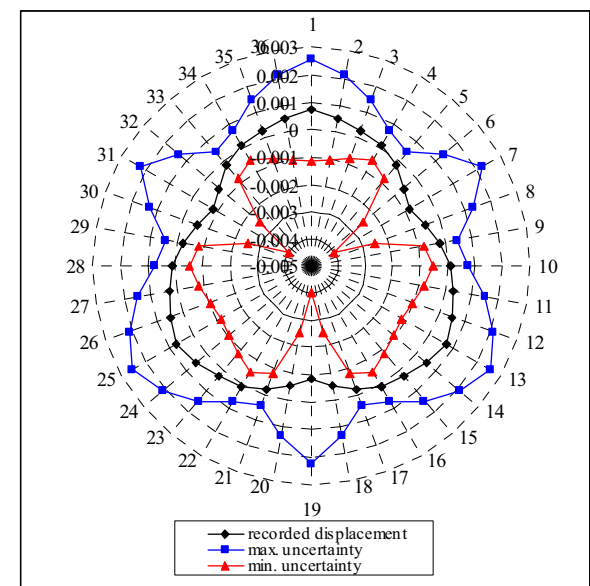

Fig. 7: Probe measurement uncertainty (stylus length $=20 \mathrm{~mm}$, latitude $=45^{\circ}$ )

\section{CONCLUSIONS}

A novel method of evaluating CMM probe measurement uncertainty with FEA modeling has been proposed and tested. It offers many advantages over other methods. It does not require a lot of experimental data and can be fast and reliable. The FEA model and its material properties can be easily changed, so that the impacts of various variables and design can be conveniently studied and optimized. Of course, during the modeling process, some information, such as material properties and component dimensions, are estimated so the modeling results will differ slightly from those obtained from the real probe operation.

\section{REFERENCES}

[1] A. Piratelli-Filho, B. Di Giacomo, "CMM Uncertainty Analysis with Factorial Design", Precision Engineering 27, 2003, p283 288.

[2] A. Weckenmann, M. Knauer, "The Influence of Measurement Strategy on the Uncertainty of CMM Measurements", Annals of the CIRP, Vol. 47, 1, 1998, p451 454.

[3] John A. Bosch, "Coordinate Measuring Machines and Systems", Marcel Dekker, Inc., 1995

[4] T. Killmaier, A. Ramesh Babu, "Genetic Approach for Automatic Detection of Form Deviations of Geometrical Features for Effective Measurement Strategy", Precision Engineering, Vol. 27, Issue 4, Oct. 2003, p370 381

[5] ISO-GUM, "Guide to the expression of uncertainty in measurement", International Organization of Standardization, Geneva, 1995.

[6] M. R. Salleh, Q. Ping Yang, Barry J., "Finite Element Modeling and Simulation of Probe System", IMTC 2005 - Instrumentation and Measurement Technology Conference, May 2005.

[7] W. Tyler Estler, et. Al., "Error Compensation for CMM Touch Trigger Probes", Precision Engineering 19, 1996, p85 97. 